\title{
An Automated Analysis of Ping-Pong Interactions in E-mail Services
}

\author{
Anne Bergeron and Jean-Christophe Manzoni \\ LACIM, Université du Québec à Montréal, \\ C.P. 8888 Succursale Centre-Ville, Montréal, Québec, \\ Canada, H3C 3P8, \\ \{anne, manzoni\}@lacim.uqam.ca
}

\begin{abstract}
Feature interactions occur when the composition of two processes produces unexpected or unwanted behaviors. The problem of detecting interactions can be formalized, but the resolution of such interactions remains almost an art since specifications must be changed in a way or another. In this paper, we describe a technique that can be used to automatically propose modifications to the original specifications in order to remove unwanted interactions. We show that this technique removes sucessfully ping-pong interactions in E-mail services where messages are endlessy duplicated by a careless user or a distribution list.
\end{abstract}

KEY-WORDS: Feature interactions, detection, resolution, E-mail services.

\section{Introduction}

Feature interactions occur when the composition of two processes produces "bad behaviors". While it is not necessarily easy to detect such bad behaviors, these can be conveniently defined as sequences of events that should not occur. Thus, the problem of defining what is feature interaction is a specification problem. For example, when processes are modelled with transition systems, one can say that a bad interaction occurs if the composition has a deadlock, or does not meet a minimal set of behaviors.

However, there is no widely accepted definition of what is feature interactions resolution [5]. One can advertise an interaction as a feature, or try to redesign the interacting processes [8], or add a supervisor that prevents bad interactions [4], [7], or even forbid the processes to interact at all. In this context, this paper proposes a technique that uses the detection phase to automatically suggest modifications to the specifications of the interacting processes. 
As a simple example, consider the two processes $P_{1}$ and $P_{2}$ modeled by the following automata.
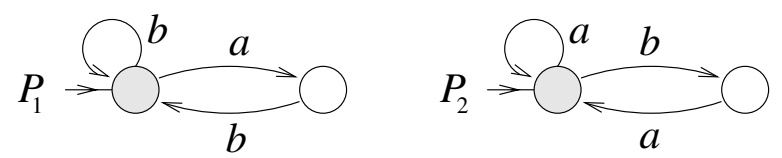

When these two processes are composed on identical events, we get:

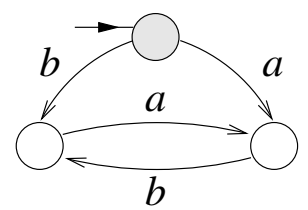

There is no possibility that this automaton returns to its initial state after event $a$ or $b$. Suppose that this is considered to be bad behavior, is there a way to modify the specifications of $P_{1}$ and $P_{2}$ such that good interactions can still occur, without deadlocking the whole process?

The central idea of this paper is to identify, in each process, transitions that should be ignored by the other. Continuing with our example, consider the following labelling of $P_{1}$ and $P_{2}$ :
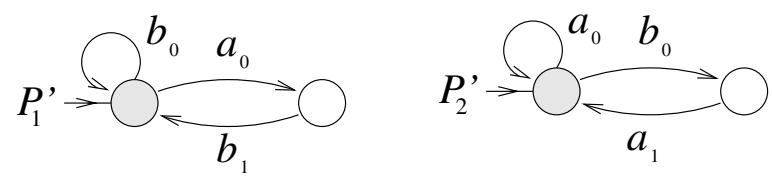

If these two processes are composed while allowing $P_{1}^{\prime}$ to ignore transition $a_{1}$, and process $P_{2}^{\prime}$ to ignore transition $b_{1}$, then we get the following composition which is deadlock free:

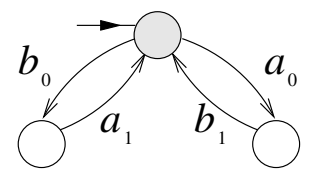

Even if this new composition seems to solve the deadlock problem, there are still many questions to answer. Can we automate this technique? What does it 
mean for a process to ignore a transition? Does this technique always remove bad interactions? Is the new specification for the processes still acceptable?

In the following sections, we will try to answer these questions using, as a running example, well-known interactions that occur in E-mail services leading to problems such as endless message duplication or ping-pong effects. Section 2 describes an abstract model of E-mail services that nevertheless captures many feature interactions, Section 3 sketches the resolution technique, and Section 4 gives an example of resolution of a non trivial problem.

\section{Modeling E-mail Services}

A basic E-mail service allows clients to exchange messages. Such a service can be augmented by features that include, for example, distribution of a message to a group of clients, or automatic response to messages. Adding these features to the basic service, especially automatic responses, can - and do - lead to eccentric behavior like endless duplication of the same message.

In order to study these behaviors, we want a model of these services that abstracts message content and delays of transmission. We will focus on exchanges of messages, assuming that if $I$ sends a message to $J$ it is received immediately, and we will model only the nature and order of exchanges.

We will consider two features: a vacation function that can be used by a client to answer automatically to its correspondents, and a mailing list. It is interesting to note that the smallest system that exhibits endless loop behavior, called the ping-pong effect, consists of only two clients with their vacation function active.

\section{$2.1 \quad$ E-mail Clients}

An E-mail client is the program used by a person sending and receiving electronic mails. Let $I$ and $J$ be two E-mail clients. Two kind of messages can be exchanged between $I$ and $J$ : $I J$ will represent a message from $I$ to $J$, and $J I$ will represent a message from $J$ to $I$.

Fig. 1 presents the two automata modeling client $I$ and client $J$.
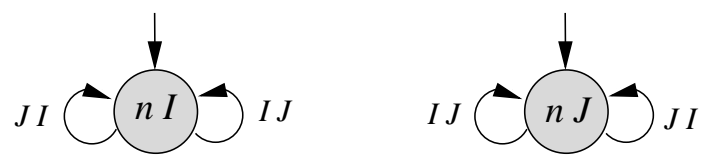

Fig. 1. Clients

Both automata have only one state, the normal state, in which ordinary electronic messages can be sent or received. 


\subsection{Vacation Function}

A vacation function is a program sending a fixed message back to any client trying to reach client $X$. For example, when going away for holiday, client $X$ might want to inform automatically every person trying to reach him of his return date. He will activate his vacation function.

Fig. 2] shows the automaton modeling an E-mail client $I$ which has activated its vacation function.

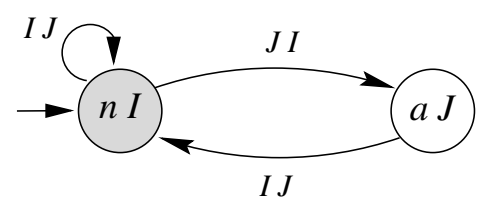

Fig. 2. Client I with its vacation function active

When a message is received, in this case event $J I$, the system goes to state $a J$ and automatically sends back a message $I J$. Note that client $I$ can still send normal messages.

The automaton for client $J$ would be similar. If there are more clients in the system, we add to the model an $a X$ state for each possible correspondent $X$.

\subsection{Mailing List}

A mailing list is a program forwarding a message received from one subscriber to every other subscriber on the list, excluding the sender. When clients $I$ and $J$ are the only subscribers to the list, we get the automaton of Fig. 3 ,

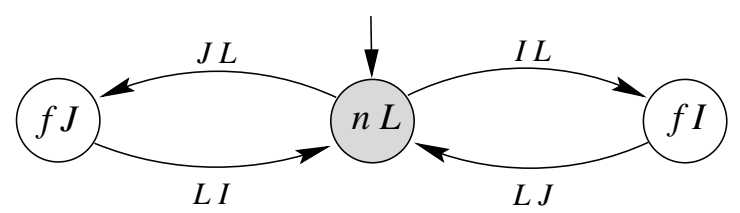

Fig. 3. A mailing list for two clients

State $f I$, for example, is reached after the reception of the message $I L$, which is a message from client $I$ to the list. It is then forwarded to each subscriber, following a loop of events that eventually comes back to state $n L$. In the case of two subscribers, a message is forwarded only to the other subscriber.

Altough the automata of Fig. 2 and 3 are relatively simple, we will see that they have a large potential for strange behaviors. In the next section, we will give 
the formal model used for composing these automata, and tools for analysing their behavior.

\section{Interaction Detection and Resolution}

\subsection{Definitions}

In this section, we consider an automaton $A$ on the alphabet $\Sigma$ to be the usual finite deterministic automaton structure 9] with associated recognized language $L_{A}$. A state $s$ is accessible if there exists a path from the initial state to $s$. We say that a state $s$ is blocking if it is accessible and there is no path from $s$ to a final state of the automaton. An automaton is blocking if it has at least one blocking state.

Let $A_{1}$ and $A_{2}$ be two automata on the alphabets $\Sigma_{1}$ and $\Sigma_{2}$ with inital states $i_{1}$ and $i_{2}$ and final states $F_{1}$ and $F_{2}$. A set $V$ of synchronizing vectors is a subset of $\Sigma_{1} \times \Sigma_{2}$. The composition $A_{1} \times_{V} A_{2}$ of two automata is an automaton on the alphabet $V$ whose states are pairs of states $\left(s_{1}, s_{2}\right)$ where $s_{1}$ is a state of $A_{1}$ and $s_{2}$ is a state of $A_{2}$. The initial state of $A_{1} \times_{V} A_{2}$ is $\left(i_{1}, i_{2}\right)$ and the final states are of the form $\left(s_{1}, s_{2}\right)$ where $s_{1}$ is in $F_{1}$ and $s_{2}$ is in $F_{2}$ (see [1]).

If $\left(a_{1}, a_{2}\right) \in V$ is a pair of event, the transition $\left(a_{1}, a_{2}\right)$ is defined in state $\left(s_{1}, s_{2}\right)$ if and only if $a_{1}$ is defined in $s_{1}$ and $a_{2}$ is defined in $s_{2}$.

As a simple example, consider the two clients of Section 2, both having activated their vacation function, with the normal state as initial and final state (Fig. 4).
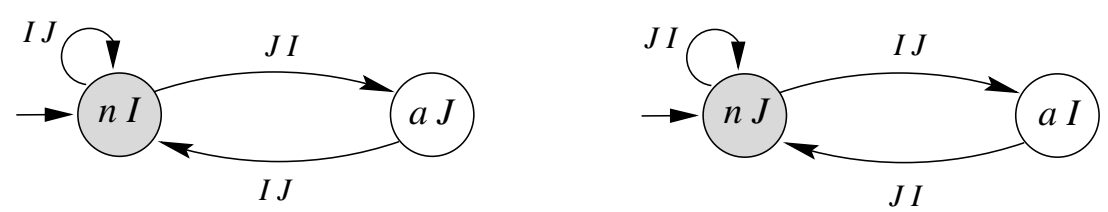

Fig. 4. Two clients to be composed

If one wants to compose these automata on identical events - that is, when a message is sent it is immediatly received - we set the synchronization vectors to:

$$
V=\{(I J, I J),(J I, J I)\} .
$$

With these vectors, we obtain the composition of Fig. 5 which is clearly blocking: client $I$ and client $J$ will exchange automatic messages forever. This is a first manifestation of the ping-pong effect.

In the sequel, we will use the possibility that a composition can be blocking, even though its factors are non-blocking, as an indication of feature interaction problems. 


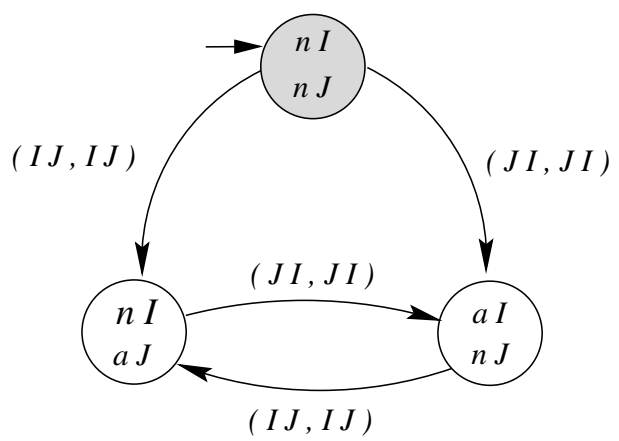

Fig. 5. A blocking automaton

Definition 1. Let $A_{1}$ and $A_{2}$ be two non-blocking automata on $\Sigma_{1}$ and $\Sigma_{2}$, and $V \subseteq \Sigma_{1} \times \Sigma_{2}$.

$A_{1}$ and $A_{2}$ are said to have unwanted feature interactions if $A_{1} \times_{V} A_{2}$ is blocking.

Using this definition, feature interaction detection can be accomplished with usual model-checking techniques. In our case, we used MEC [2], a software for analysing automata, developed at Université de Bordeaux.

Such an analysis allows one to identify all blocking states of an automaton. Once this set is known, it is possible to identify transitions that go from a nonblocking state to a blocking state. A first approach to resolution would be to inhibit those transitions, as in [7], and check the result for minimal requirements. Deciding which transitions can be inhibited is an engineering problem [3]: one has to select, for each process, which events can be inhibited or authorized. Such events are called controllable [6]. For example, in the E-mail service, a client could be prevented to send messages.

In the automaton of Fig. 5, both transitions going out of the initial state would have to be inhibited, since the two other states are blocking. This solution is clearly unacceptable since the only action that the system would be allowed to do is the null action. The specifications of the two original processes have thus to be changed. In the next section, we will describe how these specifications can be changed in order to avoid deadlocks.

\subsection{Feature Interaction Resolution}

As we saw in the last section, simply inhibiting transitions that lead to blocking states is often unacceptable. However, one could hope to remove a deadlock by rerouting some transitions between blocking states.

In order to be able to modify transitions, we first uniquely label the transitions in the blocking automaton: each copy of a transition $t$ in the product will receive a unique label $t_{i}$. If the automata are deterministics, a transition can be uniquely identified by its source and target states. Such a labeling induces 
a labeling of the two original processes. For example, Fig. 6 gives the labeling of the composition of the two client processes and the corresponding labeling of the two original processes.
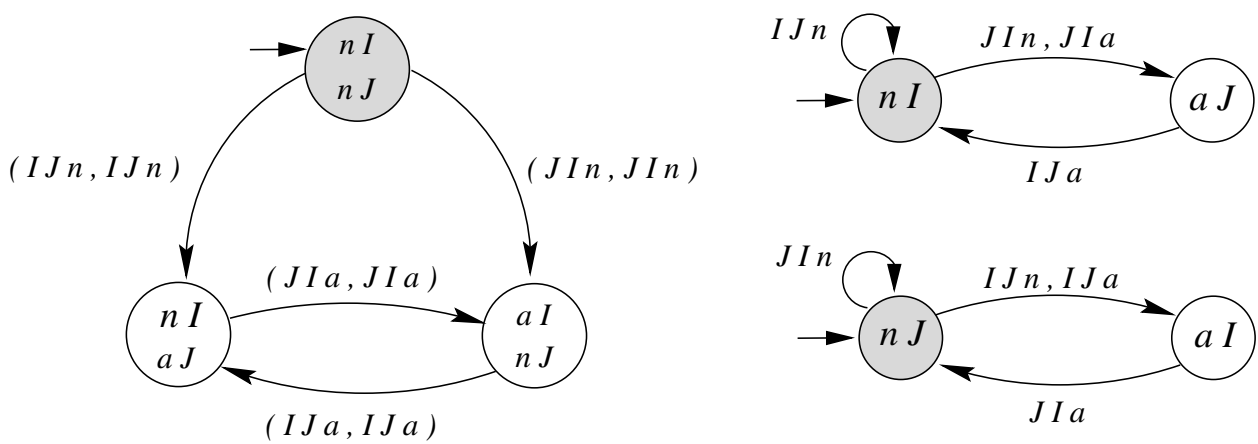

Fig. 6. Labeling the transitions

The synchronization vectors have to be changed in order to account for the labels, thus the set $V=\{(I J, I J),(J I, J I)\}$ will become

$$
V^{\prime}=\left\{\left(I J_{n}, I J_{n}\right),\left(I J_{a}, I J_{a}\right),\left(J I_{n}, J I_{n}\right),\left(J I_{a}, J I_{a}\right)\right\} .
$$

The indices $n$ and $a$ have been chosen to reflect the state of the process that emitted the message.

Our goal is to change the set $V^{\prime}$ in order that some transitions are ignored by one of the processes. This can be done by replacing a transition of the form $\left(t_{i}, t_{i}\right)$ by one of the form $\left(t_{i}, e\right)$ or $\left(e, t_{i}\right)$, where $e$ is the null event which is assumed to exist as a transparent loop on every state of the processes.

Consider, for example, the new set of transitions:

$$
V^{\prime \prime}=\left\{\left(I J_{n}, I J_{n}\right),\left(I J_{a}, e\right),\left(J I_{n}, J I_{n}\right),\left(e, J I_{a}\right)\right\}
$$

With these synchronization vectors, the composition of the two processes, as shown in Fig. 7, is non-blocking.

The impact of this modification on the original specifications is to erase some labeled transitions. This is accomplished by forcing the erased transition to loop on the state on which it was defined. In the case of the two clients, the new specifications are given in Fig. 8 .

Interpreting these new specifications in the context of E-mail services, one could say that automatic messages should be ignored by the vacation function. This new specification is acceptable since the systems retain most of their functionalities: sending messages, automatic response to non-automatic messages. In the sequel, we will use these new specifications for the vacation function. 


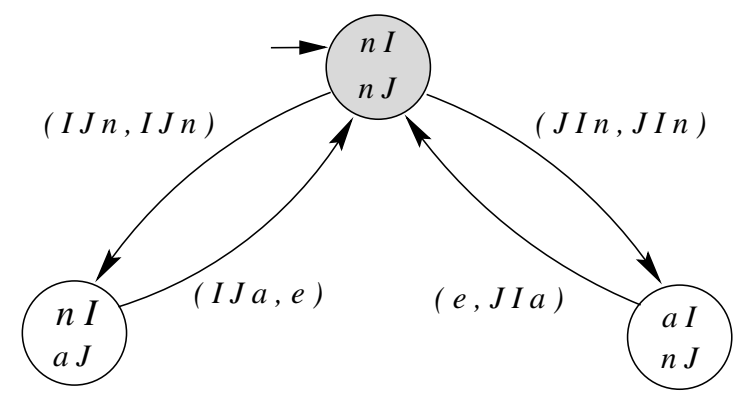

Fig. 7. A non-blocking composition
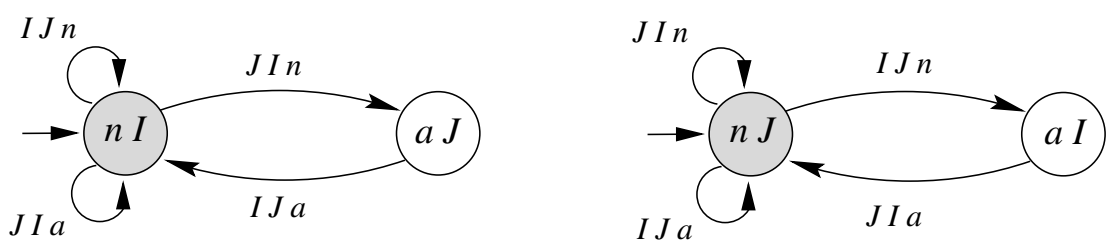

Fig. 8. New specifications

\subsection{Erasable Transitions}

We saw that deadlocks can be prevented by erasing properly selected transitions. Note that if each transition of the form $\left(t_{i}, t_{i}\right)$ is replaced by the two transitions $\left(t_{i}, e\right)$ and $\left(e, t_{i}\right)$, the communication between the two processes is effectively cut off. In that case, each process ignores the other completely, and there is no interaction, either good or bad.

We thus have to propose suitable heuristics to select which transitions are to be erased, hoping that the new processes will retain enough functionalities and communication, without deadlocking. Furthermore, we do not want to introduce deadlocks in the original specifications. To this end, we consider the following definition:

Definition 2. A transition $t$ of an automaton $A$ is essential if its removal introduces a deadlock in $A$.

We retained three criteria in order that a transition $\left(t_{i}, t_{i}\right)$ can be replaced by $\left(t_{i}, e\right)$ or $\left(e, t_{i}\right)$.

Let automata $A_{1}$ and $A_{2}$ model two processes on the same alphabet $\Sigma$, and let $V$ be a set of synchronizing vectors where $V \subseteq\{(t, t) \mid t \in \Sigma\}$. Suppose that the product $A_{1} \times_{V} A_{2}$ is blocking. After relabelling the transitions, transition $\left(t_{i}, t_{i}\right)$ can be replaced by $\left(t_{i}, e\right)$ if:

(1) Transition $t_{i}$ is not essential in automaton $A_{2}$. 
(2) Transition $\left(t_{i}, t_{i}\right)$ occurs between two blocking states of the product.

(3) There is at least one transition $\left(t_{k}, t_{k}\right)$, defined in a non-blocking state, and differing from $\left(t_{i}, t_{i}\right)$ only by its label.

The set of conditions for replacing transition $\left(t_{i}, t_{i}\right)$ by $\left(e, t_{i}\right)$ is similar.

Condition (1) ensures that the process that ignores a transition will be able to function. Condition (2) ensures that only transitions that were already problematic will be modified. Finally, condition (3) tries to keep enough communication between the processes. Indeed, if event $t$ is completely ignored by the other process, the system will probably loose important functionalities.

When all erasable transitions are erased, the resulting system can still be blocking but the technique can be iterated since the set of non-blocking states can grow.

\section{Example of Resolution: the Ping-Pong Effect.}

In Section 3, we saw that clients of an E-mail service could use their vacation function as long as it did not reply to automatic messages. We will now investigate what happens when those clients subscribe to a mailing list.

\subsection{A Message Exchange Process}

We now have three actors in the system: clients $I$ and $J$, and a list $L$. In order to model interactions between the list and the clients, we first construct a message exchange process that keeps track of the clients exchanges. Since clients $I$ and $J$ now have two correspondents, their specification has to be changed as in Fig. 9 .
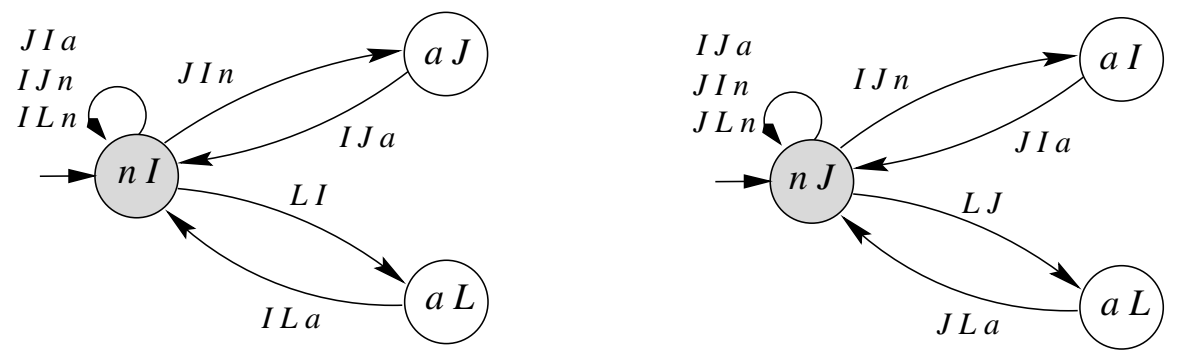

Fig. 9. Automatic responses with two correspondents

In these automata, we assume that if a message is exchanged between client $X$ and the list $L$, the other client loops on that event. Those loops have been omitted for clarity. When composing the two clients on the same events we get the message exchange automaton of Fig. 10. 


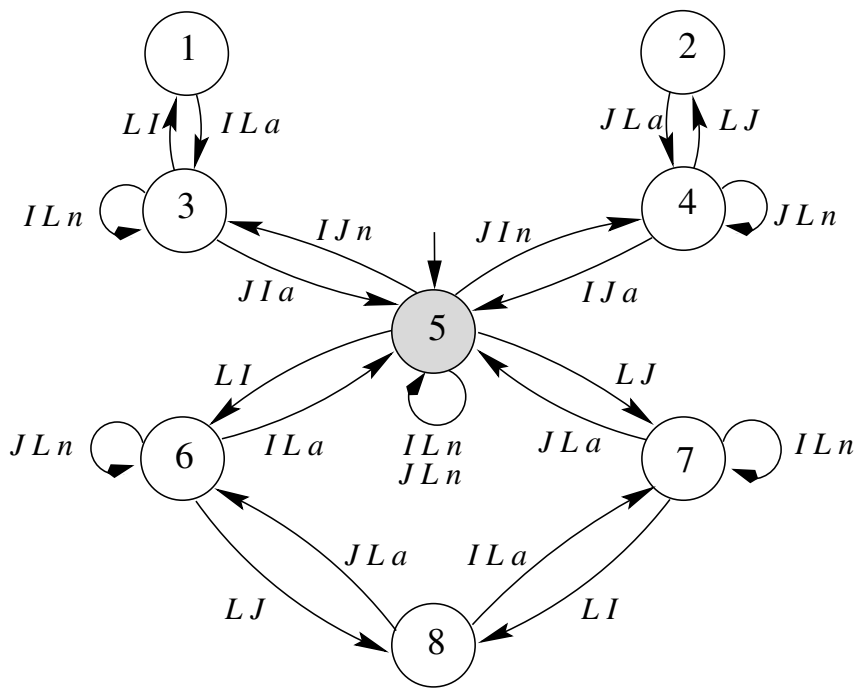

Fig. 10. Message exchange automaton

This automaton is non-blocking, and it is possible to show that it would be the case with any number of clients. The essential transitions are $I J, J I, I L$ and $J L$.

The automaton representing the mailing list, Fig. 11] is a variant of Fig. 3 of Section 2, allowing for the fact that transitions $I L$ and $J L$ have been labeled. In this automaton, transitions $L I$ and $L J$ are essential.

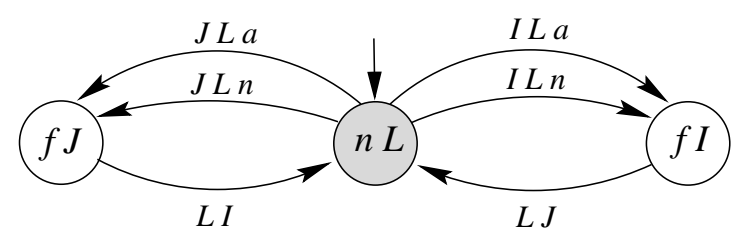

Fig. 11. Mailing list automaton

\subsection{Composing the Two Processes: The Ping-Pong Effect}

Fig. 12 shows the composition of the mailing list and the message exchange system. This automaton has only three non-blocking states, delimited by the dashed lines.

All sorts of abnormal behaviors are exhibited in this automaton. The most interesting is when, for example, client $I$ sends a message to the list in the initial state. The bold path of events following $I L n$ is a loop containing only automatic 


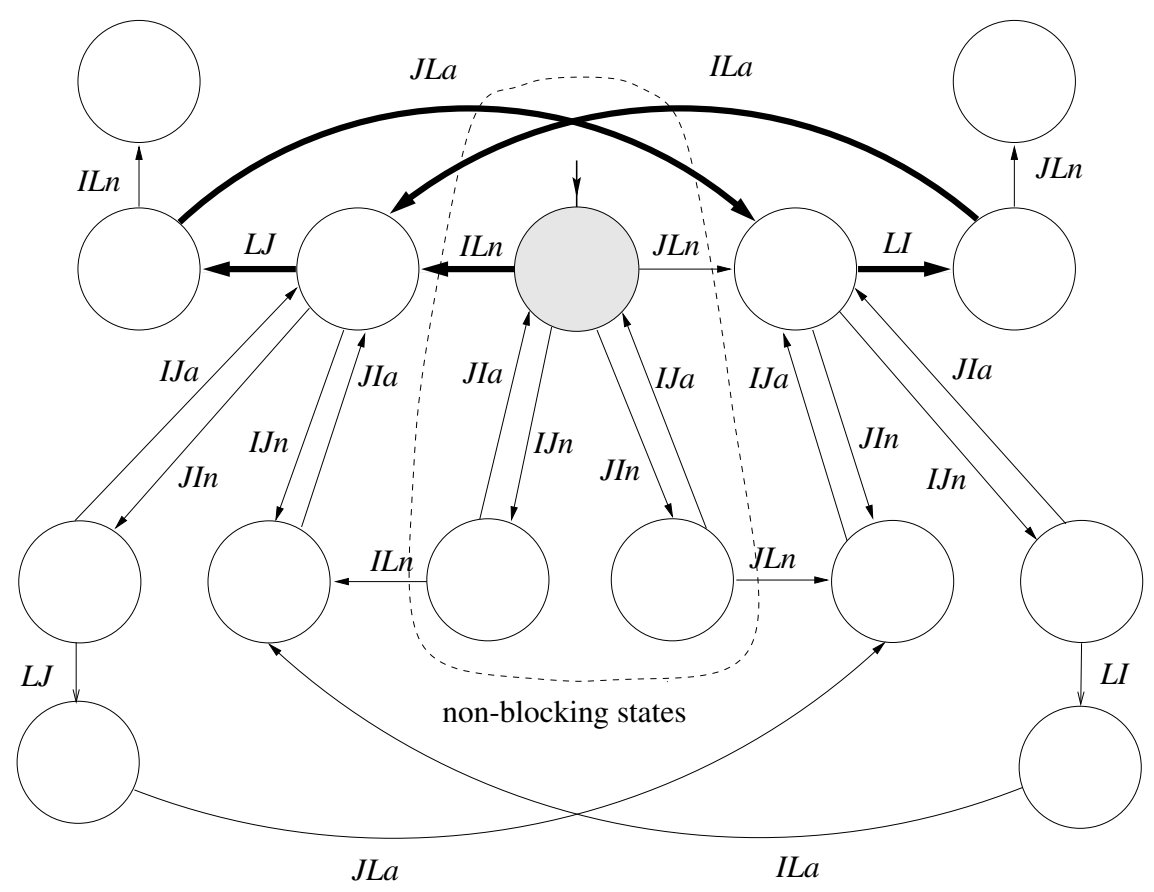

Fig. 12. The ping-pong effect

messages - LJ, JLa, LI, ILa - which will quickly flood the mail boxes of all three participants. This is a striking example of the ping-pong effect.

Another problem of this automaton is the existence of states in which no transition is defined. For instance, the sequence $I l n L J I L n$ leads to such a state. In this case, the list wants to send a message to client $J$, and client $J$ wants to send an automatic message to the list. Since neither process is in its normal state, no message can be received. One could think that the hypothesis that messages are sent and received simultaneously is too restrictive, but even if we add a buffer to the E-mail service, the problem reappears farther.

\subsection{Resolution}

We applied the algorithm described in Section 3 to the automaton of Fig. 12, erasing transitions that satisfied all three conditions of Section 3.3. After only one iteration, the new composition, shown in Fig.13, was non-blocking.

Four types of transitions were analysed:

(1) Transitions $L I$ and $L J$ were not erasable since they are not defined in any non-blocking state of Fig. 12 


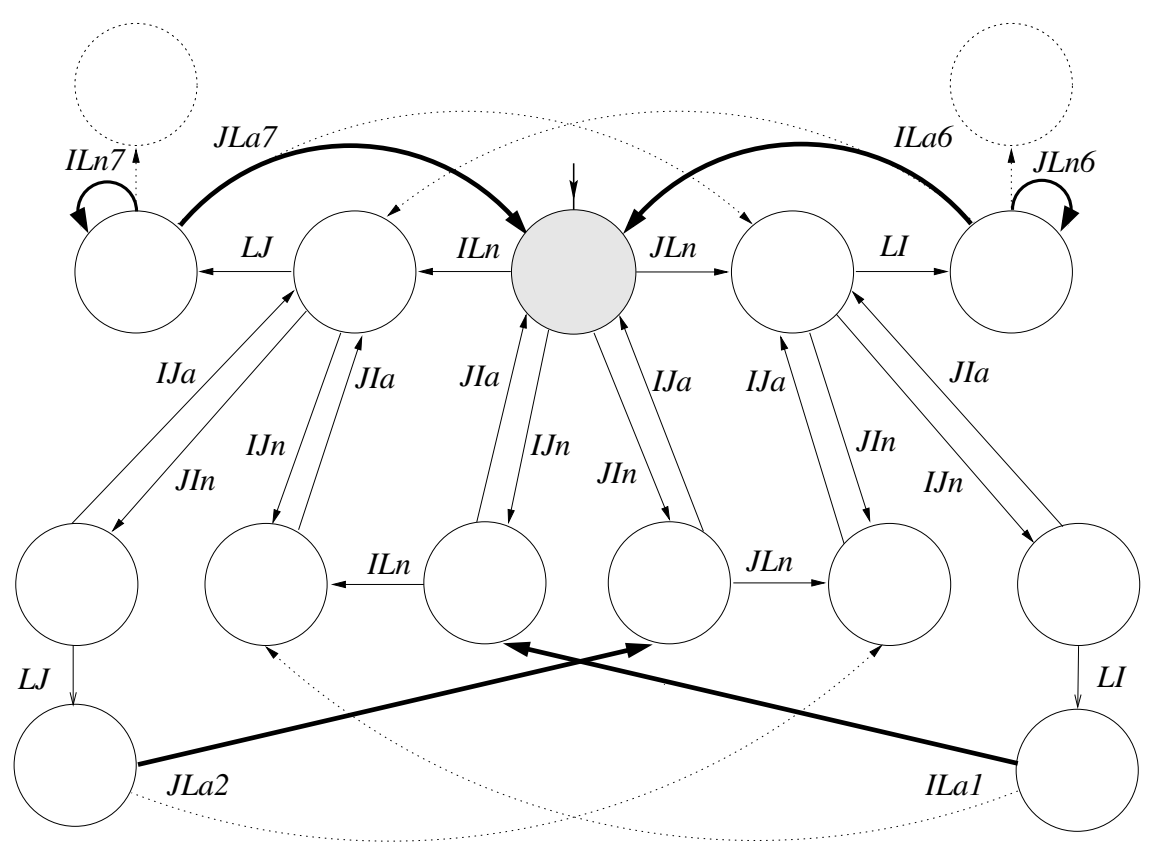

Fig. 13. The new composition

(2) All transitions of the form $I J$ or $J I$ are already ignored by the list. Erasing them does not modify the composition.

(3) Finally, only transitions that belonged to the list could be erased. The four easiest one are:

\section{ILa1, JLa2, ILa6, JLa7}

where the numbers refer to the corresponding state number of the message exchange automaton of Fig. 10 They are all automatic messages sent to the list, and should be ignored. This conclusion is similar to the one we obtained in the case of two clients with active vacation functions.

(4) Two instances of normal message also have to be ignored by the list. Those are $I L n 7$ and $J L n 6$. Those are messages sent to the list while at least one of the subscriber with active vacation function has not yet sent an automatic acknowledgement to a previous message from the list.

Thus, normal messages should not be broadcasted by the list when the exchange system is in state 6 or 7 . These states can only be reached after a first broadcast by the list, and the exchange automaton returns to state 5 after each subscriber with active vacation function has replied. Thus, if the list keeps track of the set of its absent subscribers, it can always check that all automatic re- 
sponses have been sent back before resuming its normal operations.

The revised specification for the mailing list is shown in Fig. 14.

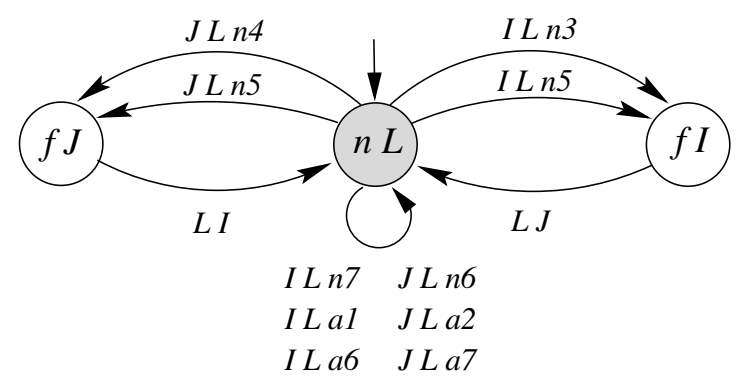

Fig. 14. The revised specification of the mailing list

\section{Conclusion}

In this paper, we proposed a technique to resolve feature interactions by automatically proposing modifications to the original specifications. The basic idea is to selectively suppress certain exchanges of information between two process in order to avoid deadlocking. We successfully applied the technique to ping-pong interactions in E-mail services.

In general it is not possible to guarantee the suppression of all deadlocks, or the meeting of minimal requirements. However, the soundness of a proposed solution can be verified with usual model-cheking techniques.

We modeled the different services at a high level of abstraction, and we were able to capture interactions with very small automata. We think that this technique could be readily applied to larger models, since the complexity of the resolution phase is linear in the number of transitions of the automaton that describes the composition of the processes.

\section{References}

1. A. Arnold, M. Nivat, Comportement de Processus, Colloque AFCET Les mathématiques de l'informatique, 1982, France, 35-68.

2. A. Arnold, Finite Transition Systems, Prentice Hall, 1994.

3. A. Bergeron, Sharing out Control in Distributed Processes, Theoretical Computer Science, 139, 1995, 163-186.

4. Y. L. Chen, S. Lafortune, F. Lin, Resolving Feature Interactions Using Modular Supervisory Control with Priorities, in Feature Interactions in Telecommunications Networks IV, IOS Press, 1997, 108-122. 
5. K. Kimbler, Adressing the interaction problem at the entreprise level, in Feature Interactions in Telecommunications Networks IV, IOS Press, 1997, 13-22.

6. P. Ramadge, W. M. Wonham, The Control of Discrete Event Processes, Proceedings of the IEEE, Vol.77 (1), 1989, 81-98.

7. J.G. Thistle, R. P. Malhamé, H.H. Hoang, S. Lafortune, Feature Interaction Modelling, Detection and Interaction: A supervisory control approach, in Feature Interactions in Telecommunications Networks IV, IOS Press, 1997, 93-107.

8. G. Utas, Feature Interactions: a software perspective, in Feature Interactions in Telecommunications Networks IV, IOS Press, 1997, 23-28.

9. D. Wood, Theory of computation, John Wiley \& Sons, New-York, 1987. 